\title{
Multilevel Programming Problems with Fuzzy Parameters: A Fuzzy Goal Programming Approach
}

\author{
Surapati Pramanik \\ Nandalal Ghosh B.T. College \\ P.O. Panpur, Narayanpur, Dist. North 24 Parganas, India, PIN-743126
}

\begin{abstract}
This paper presents fuzzy goal programming approach for solving multilevel programming problems with fuzzy parameters. The proposed approach is based on $\alpha$-cut and fuzzy goal programming. In the proposed approach, the tolerance membership functions for the fuzzily described objective functions are defined by determining individual best solution of the objective function of every decision maker. Since the objectives of the level decision makers are potentially conflicting in nature, decision deadlock may arise due to the dissatisfaction of the solution of upper level decision makers. Sometimes upper level decision makers insist to work more than stipulated working hours or overtime duty in order to meet the heavy demand of the market arising for festivals or emergency reasons. In order to survive in the open competitive market, the relaxations of lower level decision makers are very crucial for the upper level decision makers and for the organization. So in the proposed model relaxation of decision for each level decision maker is considered. The relaxation of decision is performed by providing preference bounds on the decision variables for avoiding decision deadlock. Then three fuzzy goal programming models for multilevel programming are formulated. In general, the fuzzy goal programming models offer different solutions. In order to find the best compromise solution Euclidean function is used. An illustrative numerical example is provided to demonstrate the efficiency of the proposed approach.
\end{abstract}

\section{General Terms}

Decision Making, Linear Programming, Optimization.

\section{Keywords}

Multi-level programming, Fuzzy goal programming, goal programming.

\section{INTRODUCTION}

Multi-level programming problem (MLPP) is developed to deal with hierarchical decision making problems. Burton [1], Bard and Falk [2], Anandalingam [3], Anandalingam and Apprey [4] studied different models to solve multi-level programming problems in crisp environment.

However, classical MLPP considers crisp variables and values, and produces crisp kinds of model. That is, classical MLPP does not consider randomness, vagueness or uncertainty included in the system [5]. For real world MLPP, the information available to decision makers is often imprecise due to inaccurate attribute measurements and inconsistent priority judgments. In order to deal with such situations, the concept of fuzzy set [6] is very helpful. Lai [7] at first proposed hierarchical optimization method and obtained a satisfactory solution using the concept of tolerance membership functions based on fuzzy set theory in 1996. Shih et al. [8] extended the concept of satisfactory solution of Lai
[7] using non-compensatory max-min aggregation operator for solving MLPPs. Shih and Lee [9] studied MLPPs using the compensatory fuzzy operator.

Sakawa et al. [10] investigated interactive fuzzy programming for multi-level linear programming problem. Sakawa et al. [11] used genetic algorithm to solve multi- level $0-1$ programming based on interactive fuzzy approach.

Sinha $[12,13]$ studied alternative fuzzy mathematical programming approach to solve MLPP. In Sinha's approach, last (lowest) level is most important and decision of the lowest level remains either unchanged or closest to individual best decision, which reflects the paradox that the decision power of the lowest level DM dominates the higher level decision maker. To deal such situation, Pramanik and Roy [14] studied MLPP based on fuzzy goal programming. In 2010, Baky [15] proposed FGP approach to solve multi objective MLPP. Han et al. [16] studied reference-based uncooperative multifollower tri-level decision problem based on fuzzy approach. They developed Kth-Best algorithm to find an optimal solution to the model. They presented a real-world case study on production-inventory planning.

Sakawa et al. [17] studied interactive fuzzy programming for multi-level linear programming problems with fuzzy parameters. Pramanik [18] formulated three fuzzy goal programming models for solving bi-level programming problem with fuzzy parameters extending the concept of Pramanik and Roy [19]. Pramanik and Dey [20] extended the concept of Pramanik [18] to multi-objective bi-level programming problem with fuzzy parameters where they considered relaxation provided by the both level decision makers. Pramanik et al. [21 studied decentralized bi-level multi-objective programming problem with fuzzy parameters based on fuzzy goal programming.

In the paper, the concept of Pramanik [18] is extended for multilevel programming problem.

Rest of the paper is designed as follows: Section 2 presents mathematical preliminaries. Section 3 presents formulation of FGP having fuzzy parameters. Section 4 is devoted to formulate FGP models for MLPP. In the next section 5, a numerical example is solved. In section 6 , conclusion and future direction of research are presented.

2. MATHEMATICAL PRELIMINARIES

Fuzzy set: In 1965, Zadeh [6] grounded the concept of fuzzy sets as a mathematical form for representing impreciseness.

Definition 2.1 [6] A fuzzy set $\tilde{\mathrm{A}}$ in a universe of discourse $X$ is defined by $\tilde{\mathrm{A}}=\left\{\left\langle\mathrm{x}, \mu_{\tilde{\mathrm{A}}}(\mathrm{x})\langle| \mathrm{x} \in X\right\}\right.$, where $\mu_{\tilde{\mathrm{A}}}(\mathrm{x})$ : 
$\mathrm{X} \rightarrow[0,1]$ is called the membership function of $\mathrm{A}$ and $\mu_{\tilde{n}}$

(x) is the degree of membership to which $\mathrm{x} \in \tilde{\mathrm{A}}$.

Definition 2.2 [6] Union of two fuzzy sets $\tilde{\mathrm{A}}$ and $\tilde{\mathrm{B}}$ with respective membership functions $\mu_{\tilde{\mathrm{A}}}(\mathrm{x}), \mu_{\tilde{\mathrm{B}}}(\mathrm{x})$ is defined by a fuzzy set $\tilde{\mathrm{C}}$ whose membership function is defined by $\mu_{\tilde{\mathrm{A}} \tilde{\mathrm{B}}}(\mathrm{x})=\mu_{\tilde{\mathrm{C}}}(\mathrm{x})=\max \left[\mu_{\tilde{\mathrm{A}}}(\mathrm{x}), \mu_{\tilde{\mathrm{B}}}(\mathrm{x})\right], \mathrm{x} \in \mathrm{X}$.

Definition 2.3 [6] Intersection of two fuzzy sets $\tilde{A}$ and $\tilde{B}$ with respective membership functions $\mu_{\tilde{\mathrm{A}}}(\mathrm{x}), \mu_{\tilde{\mathrm{B}}}(\mathrm{x})$ is defined by a fuzzy set $\tilde{C}$ whose membership function is defined by $\mu_{\tilde{\mathrm{A}} \cap \tilde{\mathrm{B}}}(\mathrm{x})=\mu_{\tilde{\mathrm{C}}}(\mathrm{x})=\min \left[\mu_{\tilde{\mathrm{A}}}(\mathrm{x}), \mu_{\tilde{\mathrm{B}}}(\mathrm{x})\right], \mathrm{x} \in$ $\mathrm{X}$.

Definition 2.4 $\alpha$-cut. The $\alpha$-cut of a fuzzy set $\tilde{\mathrm{A}}$ of $X$ is a non-fuzzy set denoted by ${ }^{\alpha}$ A defined by a subset of all elements $\mathrm{x} \in X$ such that their membership functions exceed or equal to a real number $\alpha \in[0,1]$, i.e. ${ }^{\alpha} \mathrm{A}=$ [ $\left.\mathrm{x}: \mu_{\sim}(\mathrm{x}) \geq \alpha, \alpha \in[0,1], \forall \mathrm{x} \in X\right]$.

\section{FORMULATION OF FUZZY GOAL PROGRAMMING HAVING FUZZY PARAMETERS}

Consider the following fuzzy optimization problem:

$\operatorname{maximize} \tilde{F}(\overline{\mathrm{x}})=\left(\tilde{\mathrm{c}}_{1} \overline{\mathrm{x}}, \tilde{\mathrm{c}}_{2} \overline{\mathrm{x}}, \ldots, \tilde{\mathrm{c}}_{\mathrm{p}} \overline{\mathrm{X}}\right)^{\mathrm{T}}$

subject to $\overline{\mathrm{x}} \in \mathrm{S}=\left\{\overline{\mathrm{x}} \in \mathfrak{R}^{\mathrm{n}} \mid \tilde{\mathrm{A}} \overline{\mathrm{x}} * \tilde{\mathrm{B}}, \overline{\mathrm{x}} \geq \overline{0}\right\}$,

where $\tilde{\mathrm{c}}_{\mathrm{p}}(\mathrm{p}=1,2, \ldots, \mathrm{P})$ are $\mathrm{n}$-dimensional fuzzy vector, $\tilde{\mathrm{B}}$ is an m-dimensional fuzzy vector, $\tilde{\mathrm{A}}$ is an $\mathrm{m} \times \mathrm{n}$ fuzzy matrix. Here, the symbol $*$ denotes respectively $\geq$, $\leq$, and $=$. $\overline{\mathrm{X}}=\left(\mathrm{x}_{1}, \mathrm{x}_{2}, \ldots, \mathrm{x}_{\mathrm{n}}\right)^{\mathrm{T}}$. Consider that the problem (2) has fuzzy coefficients having possibility distributions. Assume that $\overline{\mathrm{x}}_{\alpha}$ be a solution of (2). Here $\alpha \in[0,1]$ reflects the level of possibility at which all fuzzy coefficients is feasible.

Let $\left(\tilde{\mathrm{r}}_{\alpha}\right)$ be the $\alpha$-cut of a fuzzy number $\tilde{\mathrm{r}}$

$$
\left(\tilde{\mathrm{r}}_{\alpha}\right)=\left\{\mathrm{r} \in \operatorname{Supp}(\tilde{\mathrm{r}}) \mid \mu_{\tilde{\mathrm{r}}}(\mathrm{r}) \geq \alpha, \alpha \in[0,1]\right\}
$$

Here $\operatorname{supp}(\tilde{\mathrm{r}})$ denotes the support of $\tilde{\mathrm{r}}$. Let $(\tilde{\mathrm{r}})_{\alpha}^{\mathrm{L}}$ and $(\tilde{\mathrm{r}})_{\alpha}^{\mathrm{U}}$ be the lower bound and upper bound of the $\alpha$-cut of $\tilde{\mathrm{r}}$ respectively satisfying the following relation

$(\tilde{\mathrm{r}})_{\alpha}^{\mathrm{L}} \leq\left(\tilde{\mathrm{r}}_{\alpha}\right) \leq(\tilde{\mathrm{r}})_{\alpha}^{\mathrm{U}}$
Then, for a prescribed value of $\alpha$, for maximization-type objective function [22], $\tilde{\mathrm{F}}_{\mathrm{p}}(\overline{\mathrm{x}})(\mathrm{p}=1,2, \ldots, \mathrm{P})$ can be replaced by the upper bound of its $\alpha$-cut i.e.

$$
\left(\tilde{\mathrm{F}}_{\mathrm{p}}(\overline{\mathrm{x}})\right)_{\alpha}^{\mathrm{U}}=\sum_{\mathrm{j}=1}^{\mathrm{n}}\left(\tilde{\mathrm{c}}_{\mathrm{pj}}\right)_{\alpha}^{\mathrm{U}} \mathrm{x}_{\mathrm{j}}
$$

Similarly, for a prescribed value of $\alpha$, for minimization-type objective function [22], $\tilde{\mathrm{F}}_{\mathrm{p}}(\overline{\mathrm{x}})(\mathrm{p}=1,2, \ldots, \mathrm{P})$ is replaced by the lower bound of its $\alpha$-cut i.e.

$$
\left(\tilde{\mathrm{F}}_{\mathrm{p}}(\overline{\mathrm{x}})\right)_{\alpha}^{\mathrm{L}}=\sum_{\mathrm{j}=1}^{\mathrm{n}}\left(\tilde{\mathrm{c}}_{\mathrm{pj}}\right)_{\alpha}^{\mathrm{L}} \mathrm{x}_{\mathrm{j}}
$$

For inequality constraints

$$
\begin{aligned}
& \sum_{j=1}^{n} \tilde{A}_{i j} x_{j} \geq \tilde{B_{i}}, i=1,2, \ldots, m_{1}, \\
& \text { and } \sum_{\mathrm{j}=1}^{\mathrm{n}} \tilde{\mathrm{A}}_{\mathrm{ij}} \mathrm{x}_{\mathrm{j}} \leq \tilde{\mathrm{B}_{i}}, \quad \mathrm{i}=\mathrm{m}_{1}+1, \ldots, \mathrm{m}_{2} \text {, }
\end{aligned}
$$

can be replaced by the following constraints:

$$
\begin{aligned}
& \sum_{j=1}^{n}\left(\tilde{A}_{i j}\right)_{\alpha}^{U} x_{j} \geq\left(\tilde{B}_{i}\right)_{\alpha}^{L}, i=1,2, \ldots, m_{1} \\
& \sum_{j=1}^{n}\left(\tilde{A}_{i j}\right)_{\alpha}^{L} x_{j} \leq\left(\tilde{B}_{i}\right)_{\alpha}^{U}, i=m_{1}+1, \ldots, m_{2}
\end{aligned}
$$

For fuzzy equality constraints

$$
\sum_{j=1}^{n} \tilde{A}_{i j} x_{j}=\tilde{B_{i}}, i=m_{2}+1, \ldots, m,
$$

can be replaced by two equivalent constraints

$$
\sum_{\mathrm{j}=1}^{\mathrm{n}}\left(\tilde{\mathrm{A}}_{\mathrm{ij}}\right)_{\alpha}^{\mathrm{U}} \mathrm{x}_{\mathrm{j}} \geq\left(\tilde{\mathrm{B}}_{\mathrm{i}}\right)_{\alpha}^{\mathrm{L}} \mathrm{i}=\mathrm{m}_{2}+1, \ldots, \mathrm{m},
$$

and $\underset{j=1}{\mathrm{n}}\left(\tilde{\mathrm{A}}_{\mathrm{ij}}\right)_{\alpha}^{\mathrm{L}} \mathrm{x}_{\mathrm{j}} \leq\left(\tilde{\mathrm{B}}_{\mathrm{i}}\right)_{\alpha}^{\mathrm{U}} \quad \mathrm{i}=\mathrm{m}_{2}+1, \ldots, \mathrm{m}$,

For proof of equivalency of (11) with (12) and (13), see Lee and $\mathrm{Li}[22]$

Therefore, for a prescribed value of $\alpha$, the problem (1) reduces to the following problem:

$\operatorname{Maximize}\left(\tilde{\mathrm{F}}_{\mathrm{p}}(\overline{\mathrm{x}})\right)_{\alpha}^{\mathrm{U}}=\sum_{\mathrm{j}=1}^{\mathrm{n}}\left(\tilde{\mathrm{c}}_{\mathrm{pj}}\right)_{\alpha}^{\mathrm{U}} \mathrm{x}_{\mathrm{j}}$

subject to

$$
\begin{aligned}
& \sum_{j=1}^{n}\left(\tilde{A}_{i j}\right)_{\alpha}^{U} x_{j} \geq\left(\tilde{B}_{i}\right)_{\alpha}^{L}, i=1,2, \ldots, m_{1}, m_{2}+1, \ldots, m, \\
& \sum_{j=1}^{n}\left(\tilde{A}_{i j}\right)_{\alpha}^{L} x_{j} \leq\left(\tilde{B}_{i}\right)_{\alpha}^{U}, i=m_{1}+1, \ldots, m_{2}, m_{2}+1, \ldots, m, \\
& x_{j} \geq 0, j=1,2, \ldots, n .
\end{aligned}
$$

For simplicity, denote the system constraints (15), (16) and (17) as S. 
For a prescribed value of $\alpha$, the problem (14) reduces to a deterministic linear programming problem with multiple objectives.

The resulting membership functions for maximization-type objective functions are defined as follows:

$$
\mu_{\mathrm{p}}\left(\tilde{\mathrm{F}}_{\mathrm{p}}(\overline{\mathrm{x}})\right)_{\alpha}^{\mathrm{U}}=\frac{\left(\tilde{\mathrm{F}}_{\mathrm{p}}(\overline{\mathrm{x}})\right)_{\alpha}^{\mathrm{U}}-\left(\tilde{\mathrm{F}}_{\mathrm{p}}\right)_{\alpha}^{-}}{\left(\tilde{\mathrm{F}}_{\mathrm{p}}\right)_{\alpha}^{\mathrm{o}}-\left(\tilde{\mathrm{F}}_{\mathrm{p}}\right)_{\alpha}^{-}}, \mathrm{p}=1,2, \ldots, \mathrm{P},
$$

where the aspired level $\left(\tilde{\mathrm{F}}_{\mathrm{p}}\right)_{\alpha}^{\mathrm{o}}$ and highest acceptable level $\left(\tilde{\mathrm{F}}_{\mathrm{p}}\right)_{\alpha}^{-}$are ideal and anti-ideal solutions, respectively, which can be obtained by solving each of the following problem independently:

$$
\begin{aligned}
& \left(\tilde{\mathrm{F}}_{\mathrm{p}}\right)_{\alpha}^{\mathrm{o}}=\max _{\overline{\mathrm{X}} \in \mathrm{S}} \sum_{\mathrm{j}=1}^{\mathrm{n}}\left(\tilde{\mathrm{c}}_{\mathrm{pj}}\right)_{\alpha}^{\mathrm{U}} \mathrm{x}_{\mathrm{j}}, \mathrm{p}=1,2, \ldots, P \\
& \left(\tilde{\mathrm{F}}_{\mathrm{p}}\right)_{\alpha}^{-}=\min _{\overline{\mathrm{X}} \in \mathrm{S}} \sum_{\mathrm{j}=1}^{\mathrm{n}}\left(\tilde{\mathrm{c}}_{\mathrm{pj}}\right)_{\alpha}^{\mathrm{U}} \mathrm{x}_{\mathrm{j}}, \mathrm{p}=1,2, \ldots, P
\end{aligned}
$$

For minimization type objective function ideal and anti-ideal solutions can similarly be obtained. Assume that all of the fuzzy coefficients are trapezoidal fuzzy numbers. Trapezoidal fuzzy number $\tilde{\mathrm{r}}$ can be defined as:

$\tilde{\mathrm{r}}=\left(\mathrm{r}_{1}, \mathrm{r}_{2}, \mathrm{r}_{3}, \mathrm{r}_{4}\right)$ and the membership function of the fuzzy number will be interpreted as follows:

$$
\mu_{\tilde{r}}(\tilde{r})=\left(\begin{array}{ll}
0, & r \leq r_{1}, \\
\frac{r-r_{1}}{r_{2}-r_{1}}, & r_{1} \leq r \leq r_{2}, \\
1, & r_{2} \leq r \leq r_{3}, \\
\frac{r_{4}-r}{r_{4}-r_{3}}, & r_{3} \leq r \leq r_{4}, \\
0, & r \geq r_{4}
\end{array}\right)
$$

So, an $\alpha$-cut of $\tilde{\mathrm{r}}$ [12] (see the Fig 1) can be expressed by the following interval

$$
(\tilde{\mathrm{r}})_{\alpha}=\left[(\tilde{\mathrm{r}})_{\alpha}^{\mathrm{L}},(\tilde{\mathrm{r}})_{\alpha}^{\mathrm{U}}\right]=\left[\mathrm{r}_{1}+\left(\mathrm{r}_{2}-\mathrm{r}_{1}\right) \alpha, \mathrm{r}_{4}-\left(\mathrm{r}_{4}-\mathrm{r}_{3}\right) \alpha\right]
$$

It is to be noted that when $r^{(2)}=r^{(3)}, \tilde{r}$ reduces to the triangular fuzzy number, specified by $\left(r_{1}, r_{2}=r_{3},=r_{4}\right)$; In simple form, triangular fuzzy number $\tilde{\mathrm{r}}=\left(\mathrm{r}_{1}, \mathrm{r}_{2}, \mathrm{r}_{3}\right)$ can be defined as:

$$
\mu_{\tilde{\mathrm{r}}}(\tilde{\mathrm{r}})=\left(\begin{array}{lc}
\frac{\mathrm{r}-\mathrm{r}_{1}}{\mathrm{r}_{2}-\mathrm{r}_{1}}, & \mathrm{r}_{1} \leq \mathrm{r} \leq \mathrm{r}_{2}, \\
\frac{\mathrm{r}_{3}-\mathrm{r}}{\mathrm{r}_{3}-\mathrm{r}_{2}}, & \mathrm{r}_{2} \leq \mathrm{r} \leq \mathrm{r}_{3}, \\
0, & \text { otherwise }
\end{array}\right)
$$

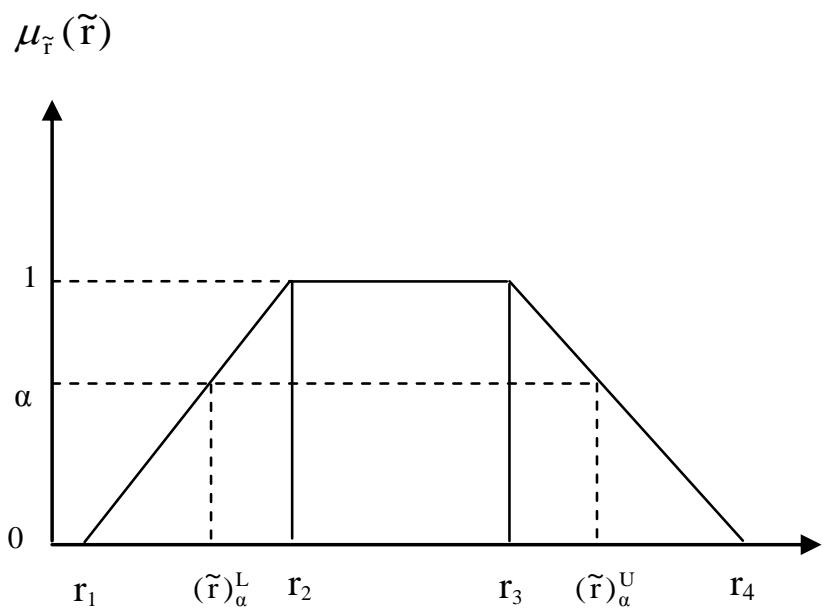

Fig1: Trapezoida fuzzy number $\tilde{\mathrm{r}}$

So, an $\alpha$-cut of $\tilde{\mathrm{r}}$ [22] (see the figure2) can be expressed by the following interval

$(\tilde{\mathrm{r}})_{\alpha}=\left[(\tilde{\mathrm{r}})_{\alpha}^{\mathrm{L}},(\tilde{\mathrm{r}})_{\alpha}^{\mathrm{U}}\right]=\left[\mathrm{r}_{1}+\left(\mathrm{r}_{2}-\mathrm{r}_{1}\right) \alpha, \mathrm{r}_{3}-\left(\mathrm{r}_{3}-\mathrm{r}_{2}\right) \alpha\right]$

and the membership function of this fuzzy number can be interpreted as follows: $(\tilde{\mathbf{r}})_{\alpha}=\left[(\tilde{\mathrm{r}})_{\alpha}^{\mathrm{L}},(\tilde{\mathrm{r}})_{\alpha}^{\mathrm{U}}\right]=\left(\mathbf{r}_{1}, \mathbf{r}_{2}, \mathbf{r}_{3}\right)$

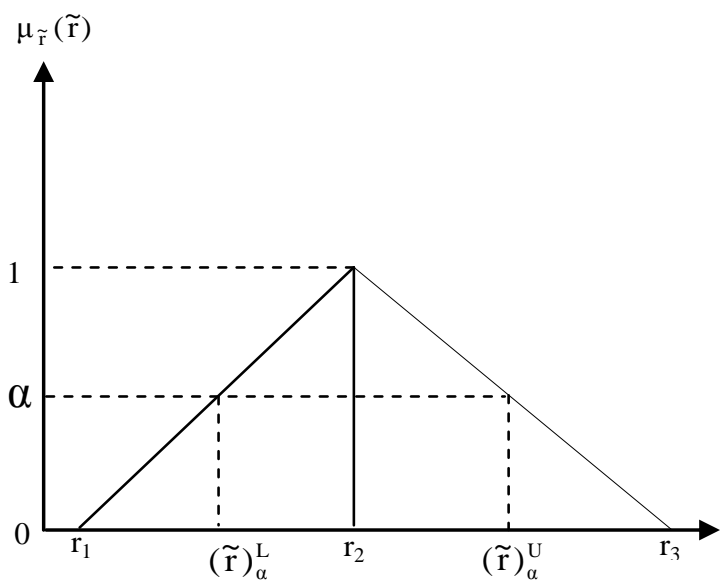

Fig 2: Triangular fuzzy number $\tilde{\mathrm{r}}$

For given value of $\alpha$, the main interest of the decision making unit is to maximize the degree of membership functions of the objective functions subject to the system constraints i.e.

$\max \mu_{\mathrm{p}}\left(\tilde{\mathrm{F}}_{\mathrm{p}}(\overline{\mathrm{x}})\right)_{\alpha}^{\mathrm{U}}, \mathrm{p}=1,2, \ldots, \mathrm{P}$,

subject to $0 \leq \mu_{\mathrm{p}}\left(\tilde{\mathrm{F}}_{\mathrm{p}}(\overline{\mathrm{x}})\right)_{\alpha}^{\mathrm{U}} \leq 1$,

$\overline{\mathrm{X}} \in \mathrm{S}$

Then according to Pramanik and Roy [14], Pramanik [18] and Pramanik and Dey [23], the FGP model for maximization type objective function can be explicitly formulated as:

FGP model (1):

$\min \lambda$

subject to

$\frac{\left(\tilde{\mathrm{F}}_{\mathrm{p}}(\overline{\mathrm{x}})\right)_{\alpha}^{\mathrm{U}}-\left(\tilde{\mathrm{F}}_{\mathrm{p}}\right)_{\alpha}^{-}}{\left(\tilde{\mathrm{F}}_{\mathrm{p}}\right)_{\alpha}^{\mathrm{o}}-\left(\tilde{\mathrm{F}}_{\mathrm{p}}\right)_{\alpha}^{-}}+\mathrm{d}_{\mathrm{p}}^{-}=1, \mathrm{p}=1,2, \ldots, \mathrm{P}$,
$\sum_{\mathrm{j}=1}^{\mathrm{n}}\left(\tilde{\mathrm{A}}_{\mathrm{ij}}\right)_{\alpha}^{\mathrm{U}} \mathrm{x}_{\mathrm{j}} \geq\left(\tilde{\mathrm{B}}_{\mathrm{i}}\right)_{\alpha}^{\mathrm{L}}, \mathrm{i}=1,2, \ldots, \mathrm{m}_{1}, \mathrm{~m}_{2}+1, \ldots, \mathrm{m}$, 
$\sum_{j=1}^{\mathrm{n}}\left(\tilde{\mathrm{A}}_{\mathrm{ij}}\right)_{\alpha}^{\mathrm{L}} \mathrm{x}_{\mathrm{j}} \leq\left(\tilde{\mathrm{B}}_{\mathrm{i}}\right)_{\alpha}^{\mathrm{U}}, \mathrm{i}=\mathrm{m}_{1}+1, \ldots, \mathrm{m}_{2}, \mathrm{~m}_{2}+1, \ldots, \mathrm{m}$,

$\mathrm{x}_{\mathrm{j}} \geq 0, \mathrm{j}=1,2, \ldots, \mathrm{n}$.

$\lambda \geq \mathrm{d}_{\mathrm{p}}^{-}, \mathrm{p}=1,2, \ldots, \mathrm{P}$,

$\mathrm{d}_{\mathrm{p}}^{-} \geq 0, \mathrm{p}=1,2, \ldots, \mathrm{P}$,

$\mathrm{d}_{\mathrm{p}}^{-} \leq 1 \geq 0, \mathrm{p}=1,2, \ldots, \mathrm{P}$

FGP model (II): $\min \zeta=\left(\sum_{\mathrm{p}=1}^{\mathrm{p}} \mathrm{w}_{\mathrm{p}}^{-} \mathrm{d}_{\mathrm{p}}^{-}\right)$

subject to the constraints given by (30), (31), (32), (34), and (35)

FGP model (III): $\min \xi=\frac{1}{\mathrm{P}} \sum_{\mathrm{p}=1}^{\mathrm{P}} \mathrm{d}_{\mathrm{p}}^{-}$

subject to the constraints given by (30), (31), (32), (34), and (35).

Using the interval expression for trapezoidal fuzzy numbers

(22), the problem (25) can be written as:

FGP model I: $\min \lambda$

subject to

$\frac{\sum_{j=1}^{n}\left[c_{p j(4)}-\left(c_{p j(4)}-c_{p j(3)}\right) \alpha\right] x_{j}-\left(\tilde{F}_{p}\right)_{\alpha}^{-}}{\left(\tilde{F}_{p}\right)_{\alpha}^{o}-\left(\widetilde{F}_{p}\right)_{\alpha}^{-}}+d_{p}^{-}=1$,

$\mathrm{p}=1,2, \ldots, \mathrm{P}$

$\left[\mathrm{A}_{\mathrm{ij}(4)}-\left(\mathrm{A}_{\mathrm{ij}(4)}-\mathrm{A}_{\mathrm{ij}(3)}\right) \alpha\right] \mathrm{x}_{\mathrm{j}} \geq \mathrm{B}_{\mathrm{i}(1)}+\left(\mathrm{B}_{\mathrm{i}(2)}-\mathrm{B}_{\mathrm{i}(1)}\right) \alpha$

$, \mathrm{i}=1, \ldots, \mathrm{m}_{1}, \mathrm{~m}_{2}+1, \ldots, \mathrm{m}$,

$\left.\left[\mathrm{A}_{\mathrm{ij}(1)}+\left(\mathrm{A}_{\mathrm{ij}(2)}-\mathrm{A}_{\mathrm{ij}(1)}\right) \alpha\right] \mathrm{x}_{\mathrm{j}} \geq \mathrm{B}_{\mathrm{i}(4)}-\left(\mathrm{B}_{\mathrm{i}(4)}-\mathrm{B}_{\mathrm{i}(3)}\right) \alpha\right]$

$, \mathrm{i}=\mathrm{m}_{1}+1,2, \ldots, \mathrm{m}_{2}, \mathrm{~m}_{2}+1, \ldots, \mathrm{m}$,

$\lambda \geq \mathrm{d}_{\mathrm{p}}^{-}, \mathrm{p}=1,2, \ldots, \mathrm{P}$,

$\mathrm{x}_{\mathrm{j}} \geq 0, \mathrm{j}=1,2, \ldots, \mathrm{n}$

$\mathrm{d}_{\mathrm{p}}^{-} \geq_{0}, \mathrm{p}=1,2, \ldots, \mathrm{P}$.

$\mathrm{d}_{\mathrm{p}}^{-} \leq 1, \mathrm{p}=1,2, \ldots, \mathrm{P}$.

Using the interval expression (22), the problem (36) and (37) can be written as follows:

FGP model II: $\min \zeta=\left(\sum_{\mathrm{p}=1}^{\mathrm{P}} \mathrm{w}_{\mathrm{p}}^{-} \mathrm{d}_{\mathrm{p}}^{-}\right)$

and

FGP model III: $\min \xi=\frac{1}{\mathrm{P}} \sum_{\mathrm{p}=1}^{\mathrm{P}} \mathrm{d}_{\mathrm{p}}^{-}$

subject to the constraints (39), (40), (41), (43), (44), and (45)

$\mathrm{w}_{\mathrm{p}}^{-}=1 /\left[\left(\tilde{\mathrm{F}}_{\mathrm{p}}\right)_{\alpha}^{\mathrm{o}}-\left(\tilde{\mathrm{F}}_{\mathrm{p}}\right)_{\alpha}^{-}\right], \mathrm{p}=1,2, \ldots, \mathrm{P}$

Using the interval expression for triangular fuzzy numbers

[24], the FGP model for maximization type objective function can be explicitly formulated as:

$\min \lambda$

subject to

$$
\frac{\sum_{j=1}^{n}\left[c_{p j(3)}-\left(c_{p j(3)}-c_{p j(2)}\right) \alpha\right] x_{j}-\left(\widetilde{F}_{p}\right)_{\alpha}^{-}}{\left(\widetilde{F}_{p}\right)_{\alpha}^{o}-\left(\widetilde{F}_{p}\right)_{\alpha}^{-}}+d_{p}^{-}=1,
$$

$\mathrm{p}=1,2, \ldots, \mathrm{P}$
$\left[\mathrm{A}_{\mathrm{ij}(3)}-\left(\mathrm{A}_{\mathrm{ij}(3)}-\mathrm{A}_{\mathrm{ij}(2)}\right) \alpha\right] \mathrm{x}_{\mathrm{j}} \geq \mathrm{B}_{\mathrm{i}(1)}+\left(\mathrm{B}_{\mathrm{i}(2)}-\mathrm{B}_{\mathrm{i}(1)}\right) \alpha$

$, \mathrm{i}=1, \ldots, \mathrm{m}_{1}, \mathrm{~m}_{2}+1, \ldots, \mathrm{m}$,

$\left.\left[\mathrm{A}_{\mathrm{ij}(1)}+\left(\mathrm{A}_{\mathrm{ij}(2)}-\mathrm{A}_{\mathrm{ij}(1)}\right) \alpha\right] \mathrm{x}_{\mathrm{j}} \leq \mathrm{B}_{\mathrm{i}(3)}-\left(\mathrm{B}_{\mathrm{i}(3)}-\mathrm{B}_{\mathrm{i}(2)}\right) \alpha\right]$

, $\mathrm{i}=\mathrm{m}_{1}+1,2, \ldots, \mathrm{m}_{2}, \mathrm{~m}_{2}+1, \ldots, \mathrm{m}$,

$\lambda \geq \mathrm{d}_{\mathrm{p}}^{-}, \mathrm{p}=1,2, \ldots, \mathrm{P}$,

$\mathrm{x}_{\mathrm{j}} \geq 0, \mathrm{j}=1,2, \ldots, \mathrm{n}$,

$\mathrm{d}_{\mathrm{p}}^{-} \geq 0, \mathrm{p}=1,2, \ldots, \mathrm{P}$.

$\mathrm{d}_{\mathrm{p}}^{-} \leq 1, \mathrm{p}=1,2, \ldots, \mathrm{P}$.

FGP model II: $\min \zeta=\left(\underset{\mathrm{p}=1}{\mathrm{P}} \mathrm{w}_{\mathrm{p}}^{-} \mathrm{d}_{\mathrm{p}}^{-}\right)$

and FGP model III: $\xi=\frac{1}{\mathrm{P}} \sum_{\mathrm{p}=1}^{\mathrm{P}} \mathrm{d}_{\mathrm{p}}^{-}$

subject to the constraints given by (50), 51), (53), (54), (55), and (56).

In Model (II), numerical weight $\mathrm{w}_{\mathrm{p}}^{-} \quad(\mathrm{p}=1,2, \ldots, \mathrm{P})$ presents the reciprocal of the admissible violation constant. The numerical weight associated with negative deviational variable represents the relative importance of achieving the aspired level of the fuzzy goal. The larger admissible violation of constants $\left[\left(\tilde{\mathrm{F}}_{\mathrm{p}}\right)_{\alpha}^{\mathrm{o}}-\left(\widetilde{\mathrm{F}}_{\mathrm{p}}\right)_{\alpha}^{-}\right]$indicates less important k-th fuzzy goal. i.e. the larger numerical weight $\mathrm{w}_{\mathrm{p}}^{-}=1 /$ $\left[\left(\widetilde{\mathrm{F}}_{\mathrm{p}}\right)_{\alpha}^{\mathrm{o}}-\left(\widetilde{\mathrm{F}}_{\mathrm{p}}\right)_{\alpha}^{-}\right],(\mathrm{p}=1,2, \ldots, \mathrm{P})$ reflects the more important of the p-th fuzzy goal.

\section{FORMULATION OF MULTILEVEL PROGRAMMING PROBLEM}

We consider a P-level programming problem of maximization-type objective function at each level. Mathematically, the problem can be stated as: $\max \tilde{\mathrm{F}}_{1}(\overline{\mathrm{x}})=\tilde{\mathrm{c}}_{11} \overline{\mathrm{x}}_{1}+\tilde{\mathrm{c}}_{12} \overline{\mathrm{x}}_{2}+\tilde{\mathrm{c}}_{13} \overline{\mathrm{x}}_{3}+\cdots+\tilde{\mathrm{c}}_{1 \mathrm{P}} \overline{\mathrm{x}}_{\mathrm{P}}$ (59) $\mathrm{x}_{1}$ $\underset{\mathrm{x}_{2}}{\max } \underset{\tilde{\mathrm{F}}_{2}}{\tilde{\mathrm{x}}_{2}}(\overline{\mathrm{x}})=\tilde{\mathrm{c}}_{21} \overline{\mathrm{x}}_{1}+\tilde{\mathrm{c}}_{22} \overline{\mathrm{x}}_{2}+\tilde{\mathrm{c}}_{23} \overline{\mathrm{x}}_{3}+\cdots+\tilde{\mathrm{c}}_{2 \mathrm{P}} \overline{\mathrm{x}}_{\mathrm{P}}(60)$

$\underset{\mathrm{x}_{\mathrm{P}}}{\max } \underset{\tilde{\mathrm{F}}_{\mathrm{P}}}{\left.\tilde{\mathrm{x}}_{\mathrm{P}}\right)}=\tilde{\mathrm{c}}_{\mathrm{P} 1} \overline{\mathrm{x}}_{1}+\tilde{\mathrm{c}}_{\mathrm{P} 2} \overline{\mathrm{x}}_{2}+\tilde{\mathrm{c}}_{\mathrm{P} 3} \overline{\mathrm{x}}_{3}+\cdots+\tilde{\mathrm{c}}_{\mathrm{PP}} \overline{\mathrm{x}}_{\mathrm{P}}(61)$ subject to

$\tilde{\mathrm{A}}_{\mathrm{i} 1} \overline{\mathrm{x}}_{1}+\tilde{\mathrm{A}}_{\mathrm{i} 2} \overline{\mathrm{x}}_{2}+\tilde{\mathrm{A}}_{\mathrm{i} 3} \overline{\mathrm{x}}_{3}+\cdots+\tilde{\mathrm{A}}_{\mathrm{iP}} \overline{\mathrm{x}}_{\mathrm{P}} \leq \tilde{\mathrm{b}}_{\mathrm{i}}$, $\mathrm{i}=1,2, \cdots, \mathrm{q}_{1}$

$\tilde{\mathrm{A}}_{\mathrm{i} 1} \overline{\mathrm{x}}_{1}+\tilde{\mathrm{A}}_{\mathrm{i} 2} \overline{\mathrm{x}}_{2}+\tilde{\mathrm{A}}_{\mathrm{i} 3} \overline{\mathrm{x}}_{3}+\cdots+\tilde{\mathrm{A}}_{\mathrm{iP}} \overline{\mathrm{x}}_{\mathrm{P}} \geq \tilde{\mathrm{b}}_{\mathrm{i}}$ $\mathrm{i}=\mathrm{q}_{1}+1, \mathrm{q}_{1}+2,, \cdots, \mathrm{q}_{2}$

$\tilde{\mathrm{A}}_{\mathrm{i} 1} \overline{\mathrm{x}}_{1}+\tilde{\mathrm{A}}_{\mathrm{i} 2} \overline{\mathrm{x}}_{2}+\tilde{\mathrm{A}}_{\mathrm{i} 3} \overline{\mathrm{x}}_{3}+\cdots+\tilde{\mathrm{A}}_{\mathrm{iP}} \overline{\mathrm{x}}_{\mathrm{P}}=\tilde{\mathrm{b}}_{\mathrm{i}}$,

$\mathrm{i}=\mathrm{q}_{2}+1, \mathrm{q}_{2}+2,, \cdots, \mathrm{m}$

$\overline{\mathrm{x}}_{1} \geq \overline{\mathrm{0}}, \overline{\mathrm{x}}_{2} \geq \overline{\mathrm{0}}, \cdots, \overline{\mathrm{x}}_{\mathrm{P}} \geq \overline{\mathrm{0}}$

$\bar{x}_{1}=\left\{x_{1}^{1}, x_{1}^{2}, \cdots, x_{1}^{n_{1}}\right\}^{\prime}:$ decision variables under the control of first level DM

$\left.\bar{x}_{2}=\left\{x_{2}^{1}, x_{2}^{2}, \cdots, x_{2}\right\}_{2}\right\}^{\prime}$ : decision variables under the control of second level DM 
$\bar{x}_{\mathrm{P}}=\left\{\mathrm{x}_{\mathrm{P}}^{1}, \mathrm{x}_{\mathrm{P}}^{2}, \cdots, \mathrm{x}_{\mathrm{P}}^{\mathrm{n}_{\mathrm{P}}}\right\}^{\prime}$ : decision variables under the control of P-th level DM.

Where' denotes transposition. $\widetilde{\mathrm{A}}_{\mathrm{i} 1},(\mathrm{i}=1,2, \ldots, \mathrm{q})$ is the fuzzy row vector of dimension $\left(1 \times \mathrm{n}_{1}\right)$. Similarly; $\tilde{\mathrm{A}}_{\mathrm{i} 2}$ are $\left(\mathrm{q}_{2}\right.$ $\mathrm{q}_{1}$ ) fuzzy row vectors, each of dimension $\left(1 \times \mathrm{n}_{2}\right)$ and $\tilde{\mathrm{A}}_{\mathrm{iP}}$ are $\left(m-q_{2}\right)$ fuzzy row vectors, each of dimension $\left(1 \times n_{P}\right)$.

$\tilde{\mathrm{A}}_{\mathrm{ip}} \overline{\mathrm{x}}_{\mathrm{P}} \quad(\mathrm{p}=1,2, \cdots, \mathrm{P})$ is the fuzzy column vector of dimension $(\mathrm{m} \times 1) . \quad \tilde{c}_{11}, \tilde{c}_{12}, \tilde{c}_{13}, \cdots, \tilde{c}_{1 \mathrm{P}}$ are fuzzy row vectors of dimension $\left(1 \times \mathrm{n}_{1}\right), \quad \tilde{\mathrm{c}}_{21}, \tilde{\mathrm{c}}_{22}, \tilde{\mathrm{c}}_{23}, \cdots, \tilde{\mathrm{c}}_{2 \mathrm{P}}$ are fuzzy row vectors of dimension $\left(1 \times \mathrm{n}_{2}\right)$ and $\tilde{\mathrm{c}}_{\mathrm{P} 1}, \tilde{\mathrm{c}}_{\mathrm{P} 2}, \tilde{\mathrm{c}}_{\mathrm{P} 3}$, $\tilde{\mathrm{c}}_{\mathrm{PP}}$ are fuzzy row vectors of dimension $\left(1 \times \mathrm{n}_{\mathrm{P}}\right)$. We take $\overline{\mathrm{x}}$ $=\overline{\mathrm{x}}_{1} \cup \overline{\mathrm{x}}_{1} \cup \cdots \cup \overline{\mathrm{x}}_{\mathrm{P}}$ and $\mathrm{n}=\mathrm{n}_{1}+\mathrm{n}_{2}+\cdots+\mathrm{n}_{\mathrm{P}}$. Here, one DM is located on each level. The problem consists of $n$ decision variables and $m$ fuzzy constraints. Decision vector $\bar{x}_{p}, p=1$, $2, \cdots, P$ is under the control of $\mathrm{p}$-th level $\mathrm{DM}$ having $\mathrm{n}_{\mathrm{p}}$ number of decision variables. For simplicity, denote the system constraints (62), (63), (64), and (65) as $\psi$.

\subsection{DETERMINISTIC FORMULATION OF MLPP WITH FUZZY PARAMETERS}

At first, we transform the fuzzily described objective functions and constraints into deterministic objective functions and constraints for a given value of $\alpha$. Now, for a given value of $\alpha$, maximization-type objective function $\widetilde{\mathrm{F}}_{\mathrm{p}}(\overline{\mathrm{x}})$ , $(p=1,2, \ldots, P)$ can be replaced by the upper bound of its $\alpha$ cut i.e.,

$$
\begin{aligned}
& \tilde{\mathrm{F}}_{\mathrm{P}}(\overline{\mathrm{x}})_{\alpha}^{\mathrm{U}}=\left(\tilde{\mathrm{c}}_{\mathrm{p} 1}\right)_{\alpha}^{\mathrm{U}} \overline{\mathrm{x}}_{1}+\left(\tilde{\mathrm{c}}_{\mathrm{p} 2}\right)_{\alpha}^{\mathrm{U}} \overline{\mathrm{x}}_{2}+\left(\tilde{\mathrm{c}}_{\mathrm{p} 3}\right)_{\alpha}^{\mathrm{U}} \overline{\mathrm{x}}_{3}+\cdots+ \\
& \left.\tilde{\mathrm{c}}_{\mathrm{pP}}\right)_{\alpha}^{\mathrm{U}} \overline{\mathrm{X}}_{\mathrm{P}}, \mathrm{P}=1,2, \ldots, \mathrm{P}
\end{aligned}
$$

The system constraints (62), (63) and (64) inequality constraints can be replaced by the following constraints:

$$
\begin{aligned}
& \sum_{\mathrm{p}=1}^{\mathrm{P}}\left(\tilde{\mathrm{A}}_{\mathrm{ip}}\right)_{\alpha}^{\mathrm{U}} \overline{\mathrm{x}}_{\mathrm{p}} \geq\left(\tilde{\mathrm{b}}_{\mathrm{i}}\right)_{\alpha}^{\mathrm{L}}, \\
& \mathrm{i}=1,2, \ldots, \mathrm{q}_{1}, \mathrm{q}_{2}+1, \mathrm{q}_{2}+2,, \cdots, \mathrm{m} \\
& \stackrel{\mathrm{P}}{\sum_{\mathrm{A}}}\left(\tilde{\mathrm{A}}_{\mathrm{ip}}\right)_{\alpha}^{\mathrm{L}} \overline{\mathrm{x}}_{\mathrm{p}} \leq\left(\tilde{\mathrm{b}}_{\mathrm{i}}\right)_{\alpha}^{\mathrm{U}}, \\
& \mathrm{i}=\mathrm{q}_{1}+1, \mathrm{~m}_{1}+2, \ldots, \mathrm{q}_{2}, \mathrm{q}_{2}+1, \mathrm{q}_{2}+2,, \cdots, \mathrm{m} .
\end{aligned}
$$

Then, for a prescribed value of $\alpha$, the MLPP reduces to the following problem.

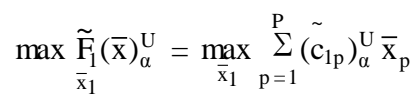

$$
\begin{aligned}
& \max \underset{\overline{\mathrm{x}}_{2}}{\tilde{\mathrm{F}}_{2}}(\overline{\mathrm{x}})_{\alpha}^{\mathrm{U}}=\max _{\overline{\mathrm{x}}_{2}} \sum_{\mathrm{p}=1}^{\mathrm{P}}\left(\tilde{\mathrm{c}}_{2 \mathrm{p}}\right)_{\alpha}^{\mathrm{U}} \overline{\mathrm{x}}_{\mathrm{p}} \\
& \max \underset{\overline{\mathrm{x}}_{\mathrm{P}}}{\tilde{\mathrm{F}}_{\mathrm{P}}}(\overline{\mathrm{x}})_{\alpha}^{\mathrm{U}}=\max _{\overline{\mathrm{x}}_{\mathrm{P}}} \sum_{\mathrm{p}=1}^{\mathrm{P}}\left(\tilde{\mathrm{c}}_{\mathrm{P}}\right)_{\alpha}^{\mathrm{U}} \overline{\mathrm{x}}_{\mathrm{p}}
\end{aligned}
$$

subject to

$$
\begin{aligned}
& \sum_{\mathrm{p}=1}^{\mathrm{P}}\left(\tilde{\mathrm{A}}_{\mathrm{ip}}\right)_{\alpha}^{\mathrm{U}} \overline{\mathrm{x}}_{\mathrm{p}} \geq\left(\tilde{\mathrm{b}}_{\mathrm{i}}\right)_{\alpha}^{\mathrm{L}}, \\
& \mathrm{i}=1,2, \ldots, \mathrm{q}_{1}, \mathrm{q}_{2}+1, \mathrm{q}_{2}+2, \cdots, \mathrm{m} \\
& \sum_{\mathrm{p}=1}^{\mathrm{P}}\left(\tilde{\mathrm{A}}_{\mathrm{ip}}\right)_{\alpha}^{\mathrm{L}} \overline{\mathrm{x}}_{\mathrm{p}} \leq\left(\tilde{\mathrm{b}}_{\mathrm{i}}\right)_{\alpha}^{\mathrm{U}}, \\
& \mathrm{i}=\mathrm{q}_{1}+1, \mathrm{~m}_{1}+2, \ldots, \mathrm{q}_{2}, \mathrm{q}_{2}+1, \mathrm{q}_{2}+2,, \cdots, \mathrm{m} . \\
& \overline{\mathrm{x}}_{1} \geq \overline{0}, \overline{\mathrm{x}}_{2} \geq \overline{0}, \cdots, \overline{\mathrm{x}}_{\mathrm{P}} \geq \overline{0}
\end{aligned}
$$

For simplicity, denote the system constraints (72), (73), and (74) as S.

To formulate the fuzzy goal programming model of the MLPP, the objective function $\widetilde{\mathrm{F}}_{\mathrm{p}}(\overline{\mathrm{x}})_{\alpha}^{\mathrm{U}}(\mathrm{p}=1,2, \cdots, \mathrm{P})$ would be transformed into fuzzy goals by means of assigning an aspiration level to each of them. Then, they are to be characterized by the associated membership functions by defining tolerance limits for achievement of the aspired levels of the corresponding fuzzy goals.

\subsection{CHARACTERIZATION OF MEMBERSHIP FUNCTION AND FORMULATION FGP MODELS}

In the decision making context, each level DM is interested in optimizing his or her own objective function, the optimal solution of each level DM when calculated in isolation would be considered as the best solution and the associated objective value can be considered as the aspiration level of the corresponding fuzzy goal. Let $\bar{x}_{\mathrm{p}}^{\mathrm{o}}=\left(\mathrm{x}_{1}^{\mathrm{B}}\right.$ be the best solution of the p-th level DM. It is quite natural that objective value which is equal to or larger than $\left(\tilde{\mathrm{F}}_{\mathrm{p}}^{\mathrm{U}}\right)_{\alpha}^{\mathrm{O}}=\max _{\overline{\mathrm{X}} \in \mathrm{S}} \tilde{\mathrm{F}}_{\mathrm{p}}(\overline{\mathrm{x}})_{\alpha}^{\mathrm{U}},(\mathrm{p}=$ $1,2, \ldots, P)$ should be absolutely satisfactory to the p-th level DM. In general, the individual best solutions are different due to the conflicting nature of the objectives. To obtain satisfactory solution each level decision maker should give some tolerance (relaxation) [24] and the relaxation of decision of each level DM depends on the needs, desires and practical situations in the decision making situation.

Then the fuzzy goals take the form $\tilde{\mathrm{F}}_{\mathrm{p}}(\overline{\mathrm{x}})_{\alpha}^{\mathrm{U}} \geq\left(\tilde{\mathrm{F}}_{\mathrm{p}}^{\mathrm{U}}\right)_{\alpha}^{\mathrm{O}}, \mathrm{p}=1$, $2, \cdots, P$.

The resulting membership functions for maximizationtype objective functions (see figure 3 ) can be defined as follows:

$$
\begin{aligned}
& \mu_{\mathrm{p}}\left(\tilde{\mathrm{F}}_{\mathrm{p}}(\overline{\mathrm{x}})_{\alpha}^{\mathrm{U}}\right)=
\end{aligned}
$$

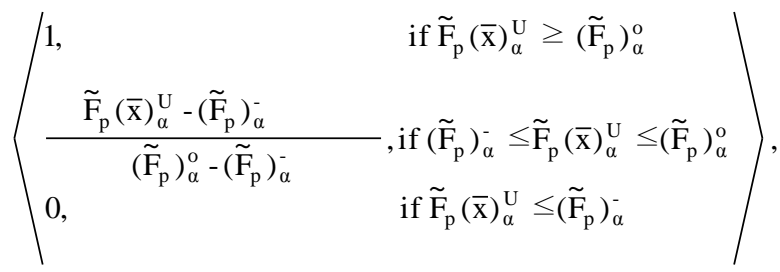

$$
\begin{aligned}
& \mathrm{j}=1,2, \ldots, P \\
& \text { Here, }\left(\tilde{\mathrm{F}}_{\mathrm{p}}^{\mathrm{U}}\right)_{\alpha}^{\mathrm{O}}=\max _{\overline{\mathrm{X}} \in \mathrm{S}} \tilde{\mathrm{F}}_{\mathrm{p}}(\overline{\mathrm{x}})_{\alpha}^{\mathrm{U}}, \mathrm{p}=1,2, \ldots, \mathrm{P} \\
& \left(\widetilde{\mathrm{F}}_{\mathrm{p}}^{\mathrm{U}}\right)_{\alpha}^{-}=\min _{\overline{\mathrm{X}} \in \mathrm{S}} \widetilde{\mathrm{F}}_{\mathrm{p}}(\overline{\mathrm{x}})_{\alpha}^{\mathrm{U}}, \mathrm{P}=1,2, \ldots, \mathrm{P}
\end{aligned}
$$




$$
\mu_{\mathrm{p}}\left(\tilde{\mathrm{F}}_{\mathrm{p}}(\overline{\mathrm{x}})_{\alpha}^{\mathrm{U}}\right)
$$

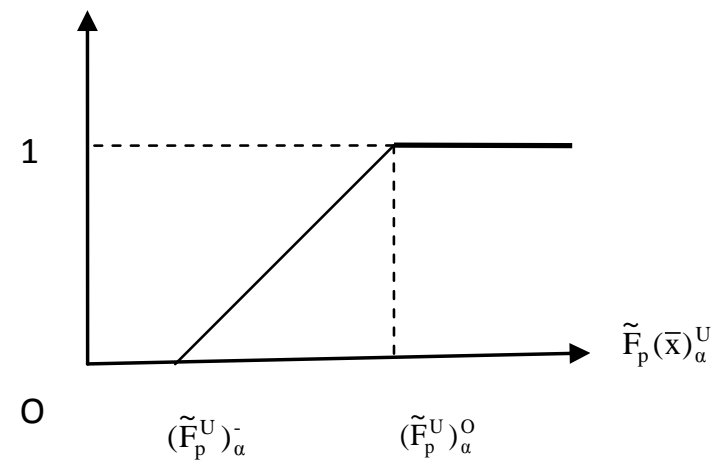

Fig 3:. Membership function for the objective function

$$
\tilde{\mathrm{F}}_{\mathrm{p}}(\overline{\mathrm{x}})_{\alpha}^{\mathrm{U}}(\mathrm{p}=1,2, \ldots, \mathrm{P})
$$

In the multi-level decision making situation, decision deadlock arises as the lower level decision makers are not satisfied with the solution of upper level decision makers. In order to deal with the deadlock situation, each level DM provides some possible relaxation on the bounds of the decision vector under his / her control. The cooperation between level DMs is useful to obtain the overall satisfactory solution.

Let $\bar{\rho}_{\mathrm{i}}^{\mathrm{L}}=\left(\rho_{\mathrm{i}}^{1 \mathrm{~L}}, \rho_{\mathrm{i}}^{2 \mathrm{~L}}, \ldots, \rho_{\mathrm{i}}^{\mathrm{n}_{\mathrm{i}} \mathrm{L}}\right)$ and $\bar{\rho}_{\mathrm{i}}^{\mathrm{U}}=\left(\rho_{\mathrm{i}}^{1 \mathrm{U}}, \rho_{\mathrm{i}}^{2}, \ldots, \rho_{\mathrm{i}}^{\mathrm{n}_{\mathrm{i}} \mathrm{U}}\right),(\mathrm{i}=$ $1,2, \ldots, \mathrm{P})$ be the vectors of preference lower and upper bounds on the decision vector $\bar{x}_{i}=\left(x_{i}^{1}, X_{i}^{2}, \cdots, \quad X_{i}^{n_{i}}\right)^{\prime}$, controlled by the $\mathrm{i}-$ th level DM $(i=1,2, \ldots, \mathrm{P})$. The $\mathrm{i}$-th level DM controls $n_{i}$ variables $\left(x_{i}^{1}, x_{i}^{2}, \cdots, x_{i}^{n_{i}}\right)$ out of $n$ variables. Suppose the best solution for the $\mathrm{i}$-th level DM is$\overline{\mathrm{x}}^{\mathrm{i} \bullet}=\left(\overline{\mathrm{x}}_{\mathrm{i}}^{\mathrm{i} \bullet}, \overline{\mathrm{x}}_{2}^{\mathrm{i} \cdot}, \ldots, \overline{\mathrm{x}}_{\mathrm{P}}^{\mathrm{i} \bullet}\right)$. The relaxation is given by the $\mathrm{i}-\mathrm{th}$ level decision as follows:

$\overline{\mathrm{x}}_{\mathrm{i}}^{\mathrm{i}^{*}}-\bar{\rho}_{\mathrm{i}}^{\mathrm{L}} \leq \overline{\mathrm{x}}_{\mathrm{i}} \leq \overline{\mathrm{x}}_{\mathrm{i}}^{\mathrm{i}^{*}}+\bar{\rho}_{\mathrm{i}}^{\mathrm{U}}$ and in general, $\bar{\rho}_{\mathrm{i}}^{\mathrm{iL}} \neq \bar{\rho}_{\mathrm{i}}^{\mathrm{iU}}, \mathrm{i}=1,2$, ..., $\mathrm{P}$.

More precisely, preference bounds of the decision variables can be presented as follows:

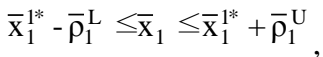

$$
\begin{aligned}
& \overline{\mathrm{x}}_{2}^{2^{*}}-\bar{\rho}_{2}^{\mathrm{L}} \leq \overline{\mathrm{x}}_{2} \leq \overline{\mathrm{x}}_{2}^{2^{*}}+\bar{\rho}_{2}^{\mathrm{U}}, \\
& \overline{\mathrm{x}}_{\mathrm{P}}^{\mathrm{P}}-\bar{\rho}_{\mathrm{P}}^{\mathrm{L}} \leq \overline{\mathrm{x}}_{\mathrm{P}} \leq \overline{\mathrm{x}}_{\mathrm{P}}^{\mathrm{P}}+\bar{\rho}_{\mathrm{P}}^{\mathrm{U}},
\end{aligned}
$$

Then the problem reduces to "

$\max \mu_{\mathrm{p}}\left(\tilde{\mathrm{F}}_{\mathrm{p}}(\overline{\mathrm{x}})_{\alpha}^{\mathrm{U}}\right), \mathrm{p}=1,2, \ldots, \mathrm{P}$

subject to

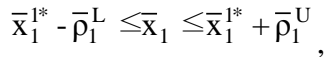

$\overline{\mathrm{x}}_{2}^{2 *}-\bar{\rho}_{2}^{\mathrm{L}} \leq \overline{\mathrm{x}}_{2} \leq \overline{\mathrm{x}}_{2}^{2^{*}}+\bar{\rho}_{2}^{\mathrm{U}}$,

$\overline{\mathrm{x}}_{\mathrm{P}}^{\mathrm{P} *}-\bar{\rho}_{\mathrm{P}}^{\mathrm{L}} \leq \overline{\mathrm{x}}_{\mathrm{P}} \leq \overline{\mathrm{x}}_{\mathrm{P}}^{\mathrm{P}}+\bar{\rho}_{\mathrm{P}}^{\mathrm{U}}$,

and $\mathrm{x} \in \mathrm{S}$
Then the three FGP models due to Pramanik and Roy [14], Pramanik [18] and Pramanik and Dey [23] can be presented in the following forms:

FGP model-1:

$\min \lambda$

subject to

$$
\begin{aligned}
& \mu_{\mathrm{p}}\left(\widetilde{\mathrm{F}}_{\mathrm{p}}(\overline{\mathrm{x}})_{\alpha}^{\mathrm{U}}\right)+\mathrm{d}_{\mathrm{p}}^{-}=1, \mathrm{p}=1,2, . ; ., \mathrm{P} \\
& \lambda \geq \mathrm{d}_{\mathrm{p}}^{-}, \mathrm{p}=1,2, . ; . ., \mathrm{P} \\
& \mathrm{d}_{\mathrm{p}}^{-} \geq 0, \mathrm{p}=1,2, . ; . ., \mathrm{P} \\
& \mathrm{d}_{\mathrm{p}}^{-} \leq 1, \mathrm{p}=1,2, . ; . ., \mathrm{P} \\
& \overline{\mathrm{x}}_{1}^{1^{*}}-\bar{\rho}_{1}^{\mathrm{L}} \leq \overline{\mathrm{x}}_{1} \leq \overline{\mathrm{x}}_{1}^{1^{*}}+\bar{\rho}_{1}^{\mathrm{U}}, \\
& \overline{\mathrm{x}}_{2}^{2^{*}}-\bar{\rho}_{2}^{\mathrm{L}} \leq \overline{\mathrm{x}}_{2} \leq \overline{\mathrm{x}}_{2}^{2^{*}}+\bar{\rho}_{2}^{\mathrm{U}},
\end{aligned}
$$

$\overline{\mathrm{x}}_{\mathrm{P}}^{\mathrm{P}}-\bar{\rho}_{\mathrm{P}}^{\mathrm{L}} \leq \overline{\mathrm{x}}_{\mathrm{P}} \leq \overline{\mathrm{x}}_{\mathrm{P}}^{\mathrm{P}}+\bar{\rho}_{\mathrm{P}}^{\mathrm{U}}$,

$\mathrm{x} \in \mathrm{S}$.

FGP model-2:

$\min \xi=\min \sum_{\mathrm{p}=1}^{\mathrm{P}} \mathrm{w}_{\mathrm{p}} \mathrm{d}_{\mathrm{p}}$

subject to

$\mathrm{d}_{\mathrm{p}}^{-} \geq 0, \mathrm{p}=1,2, . ; . ., \mathrm{P}$

$\mathrm{d}_{\mathrm{p}}^{-} \leq 1, \mathrm{p}=1,2, . ; . ., \mathrm{P}$

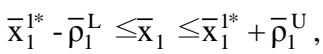

$\overline{\mathrm{x}}_{2}^{2 *}-\bar{\rho}_{2}^{\mathrm{L}} \leq \overline{\mathrm{x}}_{2} \leq \overline{\mathrm{x}}_{2}^{2^{*}}+\bar{\rho}_{2}^{\mathrm{U}}$,

$\overline{\mathrm{x}}_{\mathrm{P}}^{\mathrm{P} *}-\bar{\rho}_{\mathrm{P}}^{\mathrm{L}} \leq \overline{\mathrm{x}}_{\mathrm{P}} \leq \overline{\mathrm{x}}_{\mathrm{P}}^{\mathrm{P} *}+\bar{\rho}_{\mathrm{P}}^{\mathrm{U}}$,

$\mathrm{x} \in \mathrm{S}$.

Here, $w_{p}=\frac{1}{\left(\widetilde{\mathrm{F}}_{\mathrm{p}}\right)_{\alpha}^{\mathrm{o}}-\left(\widetilde{\mathrm{F}}_{\mathrm{p}}\right)_{\alpha}^{-}}$

FGP MODEL-3:

$$
\min \zeta=\min \frac{1}{\mathrm{P}_{\mathrm{p}=1}} \sum_{\mathrm{p}}^{\mathrm{P}} \mathrm{d}_{\mathrm{p}}
$$

\section{3 Euclidean Distance}

$\mathrm{Yu}$ [25] studied the concept of utopia point (the ideal-point) and the use the distance function for group decision analysis. Pramanik and Roy [14] used Euclidean distance to identify which FGP model provides best compromise optimal solution. Pramanik and Roy [19] also used Euclidean distance function to select appropriate priority structure in application of fuzzy goal programming technique to transportation problems. Generally, it is expected that proposed three FGP models offer three different solutions. Euclidean distance function is used to identify which FGP model provides better solution than other two FGP models. In the FGP formulation, since the aspired level of each of the membership function goals is unity, the point consisting of the highest membership value of each of the goals would represent the ideal point. The Euclidean distance [26] can be defined as follows:

$\mathrm{L}_{2}=\left[\sum_{\mathrm{j}=1}^{\mathrm{p}}\left\{1-\mu_{\mathrm{j}}\left(\mathrm{z}_{\mathrm{j}}(\mathrm{x})\right)\right\}^{2}\right]^{1 / 2}$

The solution having minimum value of $\mathrm{L}_{2}$ reflects the best compromise solution. 


\section{NUMERICAL EXAMPLE}

Consider the following MLPP with fuzzy parameters. [First Level]

$\max _{\mathrm{x}_{1}} \tilde{\mathrm{F}}_{1}(x)=\tilde{3} \mathrm{x}_{1}+\tilde{1} \mathrm{x}_{2}+\tilde{3} \mathrm{x}_{3}$

$=(2,3,4) \mathrm{x}_{1}+(1,2,3) \mathrm{x}_{2}+\left((2,3,4) \mathrm{x}_{3}\right.$

[Second Level]

$\max _{\mathrm{x}_{2}} \tilde{\mathrm{F}}_{2}(x)=\tilde{4} \mathrm{x}_{1}+\mathrm{x}_{2}-\tilde{3} \mathrm{x}_{3}$

$(3,4,5) \mathrm{x}_{1}+\mathrm{x}_{2}-(2,3,4) \mathrm{x}_{3}$

[Third Level]

$\max \tilde{\mathrm{F}}_{3}(x)=\tilde{9} \mathrm{x}_{1}-\tilde{5} \mathrm{x}_{2}+\tilde{5} \mathrm{x}_{3}$

$=(7,9,11) \mathrm{x}_{1}-(3,5,7) \mathrm{x}_{2}+(4,5,6) \mathrm{x} 3$

Subject to

$\tilde{1} \mathrm{x}_{1}+\mathrm{x}_{2}+\mathrm{x}_{3} \leq \tilde{4}$,

$\mathrm{x}_{1}-\mathrm{x}_{2}-\tilde{1} \mathrm{x}_{3} \leq \tilde{2}$,

$\mathrm{x}_{1}-\tilde{1} \mathrm{x}_{2}-\mathrm{x}_{3} \geq \tilde{1}$,

$-1 \mathrm{x}_{1}-\mathrm{x}_{2}+\mathrm{x}_{3} \leq 2$,

$x_{1} \geq 0, x_{2} \geq 0, x_{3} \geq 0$.

Here, all the fuzzy numbers are assumed to be triangular fuzzy numbers and are presented by

$\tilde{1}=(.5,1,2), \tilde{4}=(4,5,6), \tilde{2}=(1,2,3)$

By replacing the fuzzy coefficient by their $\alpha$-cuts, the MLPP can be represented as given below.

$$
\begin{array}{ll}
\max _{\mathrm{x}_{1}} & \tilde{\mathrm{F}}_{1}(\overline{\mathrm{x}})_{\alpha}^{\mathrm{U}}=(4-\alpha) \mathrm{x}_{1}+(3-\alpha) \mathrm{x}_{2}+(4-\alpha) \mathrm{x}_{3}, \\
\max _{\mathrm{x}_{2}} & \tilde{\mathrm{F}}_{2}(\overline{\mathrm{x}})_{\alpha}^{\mathrm{U}}=(5-\alpha) \mathrm{x}_{1}+\mathrm{x}_{2}-(4-\alpha) \mathrm{x}_{3}, \\
\max _{\mathrm{x}_{3}} & \tilde{\mathrm{F}}_{3}(\overline{\mathrm{x}})_{\alpha}^{\mathrm{U}}=(11-2 \alpha) \mathrm{x}_{1}-(7-2 \alpha) \mathrm{x}_{2}+(6-\alpha) \mathrm{x}_{3},
\end{array}
$$

\section{Subject to}

$(.5+.5 \alpha) \mathrm{x}_{1}+\mathrm{x}_{2}+\mathrm{x}_{3} \leq 6-\alpha$,

$\mathrm{x}_{1}-\mathrm{x}_{2}-(.5+.5 \alpha) \mathrm{x}_{3} \leq 3-\alpha$,

$\mathrm{x}_{1}-(2-\alpha) \mathrm{x}_{2}-\mathrm{x}_{3} \geq .5+.5 \alpha$,

$\left.-(.5+.5 \alpha) \mathrm{x}_{1}-\mathrm{x}_{2}+\right) \mathrm{x}_{3} \leq 3-\alpha$,

$\mathrm{x}_{1}, \mathrm{x}_{2}, \mathrm{x}_{3} \geq 0$.

For construction of membership function for specified value of $\alpha=0.5$ the upper and lower tolerance limits can be determined as follows:

$$
\begin{aligned}
\left(\tilde{\mathrm{F}}_{1}\right)_{\alpha}^{0} & =\max _{\overline{\mathrm{X}} \in \mathrm{S}} \tilde{\mathrm{F}}_{1}(\overline{\mathrm{x}})_{\alpha}^{\mathrm{U}} \\
& =\max _{\overline{\mathrm{X}} \in \mathrm{S}}(4-\alpha) \mathrm{x}_{1}+(3-\alpha) \mathrm{x}_{2}+(4-\alpha) \mathrm{x}_{3} \\
& =22.96 \\
\left(\tilde{\mathrm{F}}_{1}\right)_{\alpha}^{-} & =\min _{\overline{\mathrm{X}} \in \mathrm{S}} \tilde{\mathrm{F}}_{1}(\overline{\mathrm{x}})_{\alpha}^{\mathrm{U}} \\
& =\min _{\overline{\mathrm{X}} \in \mathrm{S}}(4-\alpha) \mathrm{x}_{1}+(3-\alpha) \mathrm{x}_{2}+(4-\alpha) \mathrm{x}_{3} \\
& =2.625
\end{aligned}
$$

Similarly, for $\alpha=0.5, \quad\left(\tilde{\mathrm{F}}_{2}\right)_{\alpha}^{0}=22.64286, \quad\left(\tilde{\mathrm{F}}_{2}\right)_{\alpha}^{-}=3.375$, $\left(\tilde{\mathrm{F}}_{3}\right)_{\alpha}^{o}=55.16,=\left(\tilde{\mathrm{F}}_{3}\right)_{\alpha}^{-}=7.5$

Level decision maker's preference bounds are considered as follows:
$2 \leq \mathrm{x}_{1} \leq 5, \quad$ ( $1^{\text {st }}$ level DM's preference bounds)

$1.25 \leq_{\mathrm{x}_{2}} \leq 3, \quad\left(2^{\text {nd }}\right.$ level DM's preference bounds)

$0.9 \leq \mathrm{x}_{3} \leq 2.5 \quad$ ( $3^{\text {rd }}$ level DM's preference bounds)

Obtained solutions are presented in the table 1 .

Table 1. Comparison between compromise optimal solutions obtained from the proposed FGP models

\begin{tabular}{|l|l|l|}
\hline $\begin{array}{l}\text { Proposed FGP } \\
\text { Model-1 }\end{array}$ & $\begin{array}{l}\text { Proposed FGP } \\
\text { Model-2) }\end{array}$ & $\begin{array}{l}\text { Proposed FGP } \\
\text { Model-3 }\end{array}$ \\
\hline $\begin{array}{l}0.2769618 \\
\mathrm{x}_{1}=4.44\end{array}$ & $\xi^{\cdot}=0.02073882$ & $\zeta^{\cdot}=0.1899187$ \\
\hline $\mathrm{x}_{2}=1.25$ & $\mathrm{x}_{1}=4.442857$ & $\mathrm{x}_{1}=4.442857$ \\
\hline $\mathrm{x}_{3}=0.92$ & $\mathrm{x}_{2}=1.267857$ & $\mathrm{x}_{2}=1.267857$ \\
\hline $\mathrm{F}_{1}=21.885$ & $\mathrm{~F}_{1}=21.86964$ & $\mathrm{x}_{3}=0.9$ \\
\hline $\mathrm{F}_{2}=18.01$ & $\mathrm{~F}_{2}=18.11071$ & $\mathrm{~F}_{2}=18.11071$ \\
\hline $\mathrm{F}_{3}=41.96$ & $\mathrm{~F}_{3}=41.77143$ & $\mathrm{~F}_{3}=41.77143$ \\
\hline$\mu_{\mathrm{F}_{1}}=0.9471$ & $\mu_{\mathrm{F}_{1}}=0.9463803$ & $\mu_{\mathrm{F}_{1}}=0.9463803$ \\
\hline$\mu_{\mathrm{F}_{2}}=0.7596$ & $\mu_{\mathrm{F}_{2}}=0.7647821$ & $\mu_{\mathrm{F}_{2}}=0.7647821$ \\
\hline$\mu_{\mathrm{F}_{3}}=0.7230$ & $\mu_{\mathrm{F}_{3}}=0.7190816$ & $\mu_{\mathrm{F}_{3}}=0.7190816$ \\
\hline $\mathrm{L}_{2}=0.37056$ & $\mathrm{~L}_{2}=0.3702941$ & $\mathrm{~L}_{2}=0.3702941$ \\
\hline
\end{tabular}

Note: Comparing Euclidean distance (see Fig 4), we see that FGP Model 2 and FGP Model 3 provide the better optimal compromise solution than FGP Model1.

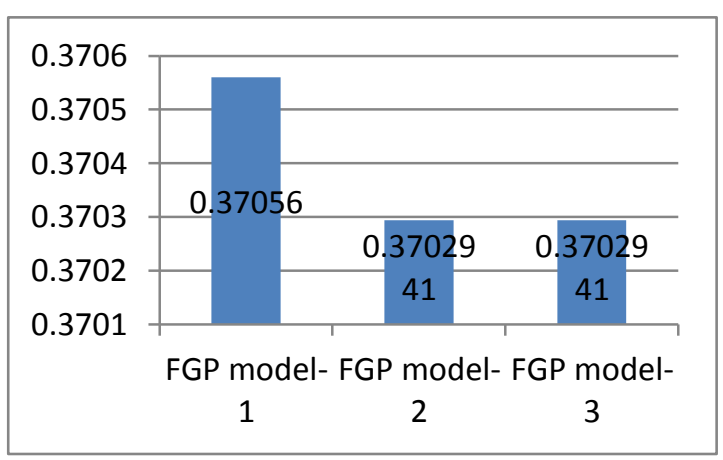

Fig 4: Comparison of Euclidean distance

\section{CONCLUSION}

In the paper, MLPP with fuzzy parameters is presented. The concept of $\alpha$-cut and goal programming are used to formulate the proposed fuzzy goal programming models. Three fuzzy goal programming models are developed and distance function is used to identify the best compromise solution. Numerical example is provided to demonstrate the proposed fuzzy goal programming models of MLPP with fuzzy parameters.

In terms of future research, the proposed approach can be extended to decentralized multi objective multi - level linear programming problem with fuzzy parameters. In addition, we will focus our future research on other relationships, such as 
cooperative and semi-cooperative situations [27], among multiple followers in decentralized MLPP with fuzzy parameters.

\section{ACKNOWLEDGMENTS}

The author would like to acknowledge the constructive comments and suggestions of the anonymous referees.

\section{REFERENCES}

[1] Burton, R. M. 1977. The multilevel approach to organizational issues of the firm. Omega 5, 457-468.

[2] Bard, J. F. and Falk, J. E. 1982. An explicit solution to the multi-level programming problems. Computers and Operations Research 9 (1), 77-100.

[3] Anandalingam, G. 1988. A mathematical programming model of decentralized multi-level systems. Journal of the Operational Research Society 39 (11), 1021-1033.

[4] Anandalingam, G. and Apprey, V. 1991. Multilevel programming and conflicting resolution. European Journal of Operational Research 51, 233-247.

[5] Watada, J., Wang, S., and Pedrycz, W. 2009. Building confidence-interval-based fuzzy random regression model. IEEE Transactions on Fuzzy Systems.11 (6), $1273-1283$.

[6] Zadeh, L. A. 1965 Fuzzy sets, Information and Control 8, 338-353.

[7] Lai, Y. J. 1996. Hierarchical optimization: a satisfactory solution. Fuzzy Sets and Systems 77, 321-335.

[8] Shih, H.S., Lai, Y.J., and Lee, E.S. 1996. Fuzzy approach for multi-level programming problems. Computers \& Operations Research 23 (1), 73-91.

[9] Shih, H.S. and Lee, E.S. 2000. Compensatory fuzzy multiple level decision making. Fuzzy Sets and Systems 114 (1), 71-87.

[10] Sakawa, M., Nishizaki, I., and Uemura, Y. 1998. Interactive fuzzy programming for multilevel linear programming problems. Computers and Mathematics with Applications 36 (2), 71-86.

[11] Sakawa, M., Nishizaki, I., and Hitaka, M. 1999. Interactive fuzzy programming for multi-level 0-1 programming through genetic algorithms. European Journal of Operational Research 144(3), 580 - 588.

[12] Sinha, S. 2003. Fuzzy mathematical programming applied to multi-level programming problems. Computers and Operations Research 30 (9), 1259 - 1268.

[13] Sinha, S. 2003. Fuzzy programming approach to multilevel programming problems. Fuzzy Sets and Systems $136(2), 189-202$

[14] Pramanik, S. and Roy, T. K. 2007. Fuzzy goal programming approach to multi-level programming problem. European Journal of Operational Research 176(2), 1151-1166

[15] Baky, I. A. 2010. Solving multi-level multi-objective linear programming problems through fuzzy goal programming approach. Applied Mathematical Modelling 34 (9), 2377-2387.

[16] Han, J. Lu, J., Hu, Y., and Zhang G. 2015. Tri-level decision-making with multiple followers: model, algorithm and case study. Information Sciences 311, 182-204.

[17] Sakawa, M., Nishizaki, I. and Uemura, Y. (2000). Interactive fuzzy programming for multi-level linear programming problems with fuzzy parameters. Fuzzy Sets and Systems. 109: 3-19.

[18] S. Pramanik. 2012. Bilevel programming problem with fuzzy parameter: a fuzzy goal programming approach. Journal of Applied Quantitative Methods. 7(1), 09-24.

[19] Pramanik, S. and Roy, T. K. 2008. Multiobjective transportation model with fuzzy parameters: based on priority based fuzzy goal programming approach. Journal of Transportation Systems Engineering and Information Technology 8(3), 40-48.

[20] S. Pramanik, Dey, P. P. 2011. Bi-level multi-objective programming problem with fuzzy parameters. International Journal of Computer Applications, 30 (10) 13-20.

[21] S. Pramanik, Dey, P. P., and Giri, B. C. 2011. Decentralized bilevel multiobjective programming problem with fuzzy parameters based on fuzzy goal programming. Bulletin of Calcutta Mathematical Society. 103 (5), 381-390.

[22] Lee, E. S., and Li, R. J. 1993.Fuzzy multiple objective programming with Pareto optimum. Fuzzy Sets and Systems 53,275-288.

[23] Pramanik, S., Dey, P. P. 2011. Quadratic bi-level programming problem based on fuzzy goal programming approach. International Journal of Software Engineering and Applications 2(4), 41-59.

[24] Dey, P. P., Pramanik, S. , and Giri, B.C. 2014. TOPSIS approach to linear fractional bi-level MODM problem based on fuzzy goal programming. Journal of Industrial and Engineering International 10(4), 173-184.

[25] Yu, P.L. 1973. A class of solutions for group decision problems. Management Science 19, 936-946.

[26] Zeleny, M. 1982. Multiple criteria decision making. McGraw-Hill, New York.

[27] Lu, J., Zhang, G. , Montero, J., and Garmendia, L. 2012. Multifollower trilevel decision making models and system. IEEE Transactions on Industrial Informatics 8(4), 974-985. 